\title{
Development of the GEA within the G-to-G system of the Iranian e-government: Views, experiences, and visions
}

\author{
Behrouz Zarei, Hosseinn Sharifi \\ Behrouz Zarei \\ Management School, Tehran University, Gisha, Tehran, Iran \\ , Tel: +982188003575 bzarei@ut.ac.ir \\ Hosseinn Sharifi: \\ University of Liverpool Management School, Chatham Building, \\ University of Liverpool, PO Box 147, Liverpool L69 7ZH, UK, Tel.: 0044- \\ 151-7953622, Fax.: 0044-151-7953666, \\ sharifi@liv.ac.uk
}

\begin{abstract}
Acknowledging the necessity of utilizing the new electronics, information and communication technologies the movement toward implementation of e-government in Iran has recently received the attention of the authorities and policy makers. Achieving this end needs first providing a clear definition for egovernment in Iran, to encompass its specific cultural, social and political characteristics, and also its actual and potential grounds for science and technology. It is argued that Government to Government (G-to-G) as a major part of the e- government consists of various components including the Government Electronic Administration (GEA) which will provide foundation required to monitor and support G-to-G system in particular and to enhance the capacity of government policy making in general. This paper addresses the efforts made toward development and implementation of Iran's GEA, the experiences gained, and the visions and views on its future developments.
\end{abstract}




\section{Introduction}

Internet has changed the way organizations, communities, and individuals work, learn and interact. Governments regardless of their political systems realize the necessity and importance of modernization in order to meet requirements of global competitiveness. E-government is one of the alternatives for this modernization undertaken by many counties. It has attracted the attention of politicians, scientists, and statesmen of the world in the recent years and many countries have devoted considerable efforts and resources for its implementation (Hwang et al, 1999; Slaton \& Becker, 2000; Wimmer 2002).

Academics have suggested various definitions for e-government. Whitson and Davis (2001) have defined e-government as "implementing cost-effective models for citizens, industry, federal employees, and other stakeholders to conduct business transactions online". Tapscott (1996) defined e-government as an "inter-networked government", and Nadler and Tushman (1997) emphasised that technology is only "one of the structural materials". Sprecher (2000) considers e-government as a technology to help simplify and automate transactions between governments and constituents, businesses, or other governments. Burn and Robins (2003) explain egovernment as governments' efforts to provide citizens with the information and services they need, using a range of information and communication technologies. Luling (2001) defines e-government as "online government services, that is, any interaction one might have with any government body or agency, using the Internet or the World Wide Web', Taking a more comprehensive view, Aicholzer and Schmutzer (2000) saw "e-government covering changes of governance in a twofold manner: (1) transformation of the business of governance, i.e. improving service quality delivery, reducing costs and renewing administrative processes; (2) transformation of governance itself, i.e. re-examining the functioning of democratic practices and processes".

E-government can be classified from various aspects. For instance from the view point of delivered services, it can be divided to delivering electronic services to citizens and businesses with the aim of providing online services, electronic democracy with the aim of setting up the electronic polling, and electronic administration with the aim of providing support for policy and decision makers and facilitating team works with operational and experts levels. E-government may be divided into three major elements from the perspective of interactions between government, business, and citizens: Firstly, Government to Government communication (G-to-G), including agencies intercommunication, and communications between the governments. It also includes, as its sub-system, the Government Electronic Administration (GEA) for facilitating and supporting the process of policy making and administration for the country through application of information and communication technology (ICT). Secondly, Government agencies' communication with citizens (G-to-C) including government and all if its affiliated 
agencies communication with citizens in non-commercial (public) sector. Thirdly, Government agencies communication with Business sector (G-to-B).

Although, all these elements share many common aspects, they are different in terms of objectives and key issues. For instance, in G-to-B, issues such as electronic payment is of high importance while for citizens, availability and access to such communications for prevention of information gap is of more importance. In G-to-G, issues such as improvement of processes and quality of decisions are critical. However, issues such as security are particularly emphasized in all functions.

Despite so many efforts to clarify the e-government, various countries use this term to express different concepts. For example, USA employs this concept to facilitate easier access to government information and services via modern ICT technologies, and to improve the quality of services leading to public satisfaction (egovernment strategy). China's definition follows the US, except for the promotion of democratic participation (Zhang, 2001). In New Zealand it is used as a means for automated communication inside the government and government to public, enabling citizens to receive needed information, establish interactive services, and to have cheap, easy and effective interactions with their representatives (Jenkins, 2002). Other countries have also interpreted this term differently, but it is recognized as a key strategy to attain competitive advantage in the twenty first century and a core means for reforming the governments. There is, however, a common theme among all these interpretations which is to set the orientation of all sectors of the society on a customer-based direction.

Hence, from a conceptual point of view, e-government can be considered as a major weapon for leaping to a new performance level (break through performance as opposed to incremental improvement), including reducing the operations cycle time, responding to impatient and hasty citizens in receiving quality, low cost and immediate services, and also satisfying the government staff who themselves suffer from the shortcomings of the current administration systems. Also e-government can be accounted as a tool for improving decision-making and a totally new way of thinking out of which change of processes, providing online services, and providing convenient services to citizens could be expected. It should be argued, thus, that only the use of new information technologies in inefficient organisations cannot be considered as e-government, since it has reverse effects by magnifying pitfalls and creating new and more problems.

The next section of the paper briefly discusses the history of G-to-G in Iran, followed by elaborating its concepts, requirements, and dimensions and how GEA has emerged. Section 4 then focuses on GEA benefits and services followed by introduction of main components of GEA and finally, reviews the implementation challenges in the Iranian environment. 


\section{Background of G-to-G system in Iran}

Although, the concept of e-government is relatively new in the literature of Iranian administration and management, the application of information technology to enhance the efficiency of operations, and implementation of Executive Management Information Systems dates back to more than fifteen years ago. Some attempts in this regard can be highlighted as follows:

- 1984-5: Networking Information management system of the Prime Minister office with a few major organisations using mainframe systems.

- 1992: Replacing personal/mini computers with mainframe systems. Applications such as the Cabinet's meeting information system were set up.

- 1993: Developing information systems for monitoring national projects with high priority, controlling essential processes, and installation of the President's MIS.

- 1993-4: Conducting extensive information needs analysis for the government which led to the recognition of a private communication network, later called Government Network. As a result it was also concluded that government agencies lacked access to sufficient, timely, structured, and coherent information, and in some cases they tend to resist sharing their information.

- 1995: Implementation of IT-based solutions in the process of verification and decision-making on the proposals referred to the Cabinet. Also efforts were made to study and understand the applicability of decision models in the Cabinet's meetings, and also finding ways to change the passive attitude of the Cabinet in response to the proposals. As a result it was concluded that access to the information distributed in different agencies is vital to the government and the Cabinet.

- 1997: Installing an experimental private network with the prime goal of safe and quality communication between Ministries and the Cabinet.

- 2001: Communication between Ministers and Cabinet proved insufficient, and the need for extension to other levels of government, whose information was decisive for the cabinet, was identified. As the result, in 2001 the government network plan (now known as GEA) was initiated which is intended to play as the infrastructure of the Iranian G-to-G. The network having born out of a need, and not merely an imitation, is expected to enjoy a natural growth and keep its efficiency until the need is satisfied.

G-to-G system of Iran, originated from inside the government's administration area as its commencing point and heart, has been defined around three axes: Needs, Problems, and Possibilities/Facilities. Needs have played the most important role in the formation of the Iranian G-to-G. The Cabinet and the President office's needs for information produced in government agencies, from one side, and the agencies' real need for digitised information services at national, regional and international levels 
from the other side, are the most important factors justifying the G-to-G. In addition, problems such as inefficient management of operations, lengthy government decision processes and dissatisfied customers, and waste of scarce resources have been important factors leading to the realisation of the need for G-to-G. Also, available facilities, and managerial, cultural, and financial restrictions have been effective in shifting the idea of e-government into what is now presented as G-to-G and GEA.

\section{Concepts, requirements, and dimensions of the Iranian G-to-G}

The main objective of the G-to-G system is to digitize the internal operations and communications of the government organizations and staff. It endeavours to improve government performance quality through elimination of agencies' physical boundaries, information sharing, strengthening the organisations, reducing costs and expenses, and improving the effectiveness of the management systems. It also includes information flow within domestic organizations as well as communication with foreign governments. Therefore, conceptually, G-to-G is central ring of linking strategy, process, organization, and technology so as to provide effective procedures to perform timely operations, and make more information available easier. In other words, G-to-G can be defined as a way for fundamental transformation of government through providing timely services (NZ E-Commerce Strategy, 2000), and as a comprehensive model which can be used to reinvigorate the government's operations processes (Sprecher, 2000).

The G-to-G, as a tool for coordinating various sections of the government, was identified as the most significant element among other elements of the Iranian egovernment. Main reasons supporting the choice of G-to-G are as follows:

Ineffectiveness of the government's body: Successful implementation of egovernment requires effective coordination of the government with citizens and businesses through ICT applications. This, however, must be led by enhancing the efficiency and effectiveness of the government's administration system itself. Otherwise, the result will be further deterioration in the satisfaction of two other sectors. The significance of this point becomes more apparent considering the fact that Iranian government plays the central role in the initiation and implementation of major functions in Iran. Therefore, it will be essential to view the G-to-G system as the medium to renovate the government structure and improve its effectiveness.

Controllable nature of the government: Building up the e-government requires extensive integration of functions and activities which needs intense planning and control. The centralised nature of the Iranian government provides grounds for this purpose, while other two elements of the e-government are much less controllable.

Larger share of government in the national economy: The government owns and controls the major part of the national wealth and resources and, therefore, 
considerable improvements shall be expected from the development of the G-to-G system.

Dependence of other e-government elements $(G-t o-B / C)$ on $G$-to- $G$ : Other elements of the e-government will fail in the absence of a well-organised and tuned G-to-G. In other words, the preparation of public and business sectors for joining and using the e-government will not lead to the expected results if the government is not prepared for this purpose.

Motivated organizations with low readiness: The penetration of the ICT culture in the country, and hence government agencies, has attracted the interest of government agencies to use IT solutions. This willingness can be considered as an opportunity. However, the risk associated with adopting this technology without careful plans could cause further inconsistency, redundancy, and waste of resources if not properly directed. For example, Finland experiences show that failing to observe this opportunity can impose extra expenses (E-Government Strategy). This issue is a strong reason for organizing these efforts in the G-to-G framework.

$G$-to- $G$ and $G E A$ being situated on the critical path of the e-government: The government status-quo seems to be vast and complex. For example, business processes of the government are massive in number and complicated in structure, with extensive interactions with other processes. Most of these processes are interdepartmental, many of which are rusty and out of form. Therefore, the development of G-to-G system will be a highly time consuming process, while it is expected to be much less in other elements of the e-government due to their smaller size and limited domains. This means that establishment of the Iranian e-government has to start from building the G-to-G system.

Alignment of the G-to-G with the government policy-making strategies: Success of recent government policies in reducing its realm of ownership and responsibility, and playing its logical role as the policy maker, entails strong tools and also effective means to ensure achieving the projected policies. Failure of traditional methods and approaches has widely been experienced in the past. It is clear that without access to accurate and timely information and in the absence of powerful control tools, achieving the government's goals would be somehow impossible. Establishment of the Iranian G-to-G system will play a key role in assisting the government to accomplish this task.

Creating competitive advantage for the country: In terms of international competition, achieving a sustainable competitive advantage in various political and economic dimensions requires a modern, flexible, efficient and effective government. Realisation of these characteristics is subject to the government's ability in rapid and accurate decision-making, and intelligent and sensible diplomacy at international level. The G-to-G system (and GEA in the centre) will contribute to this objective.

The position of GEA in connection with other elements of the e-government and also with its main users is shown in figure 1 . On the left side of the figure, the interaction of G-to-G with other sections of an information community is 
demonstrated. The largest circle represents the national communication network. Within this network, e-government is situated in its broadest view which includes all interactions with business, citizens, and foreign governments. Within this section, is situated GEA as a component of G-to-G which targets the top administration of the country. Four main groups of GEA users are also shown on the right pyramid.

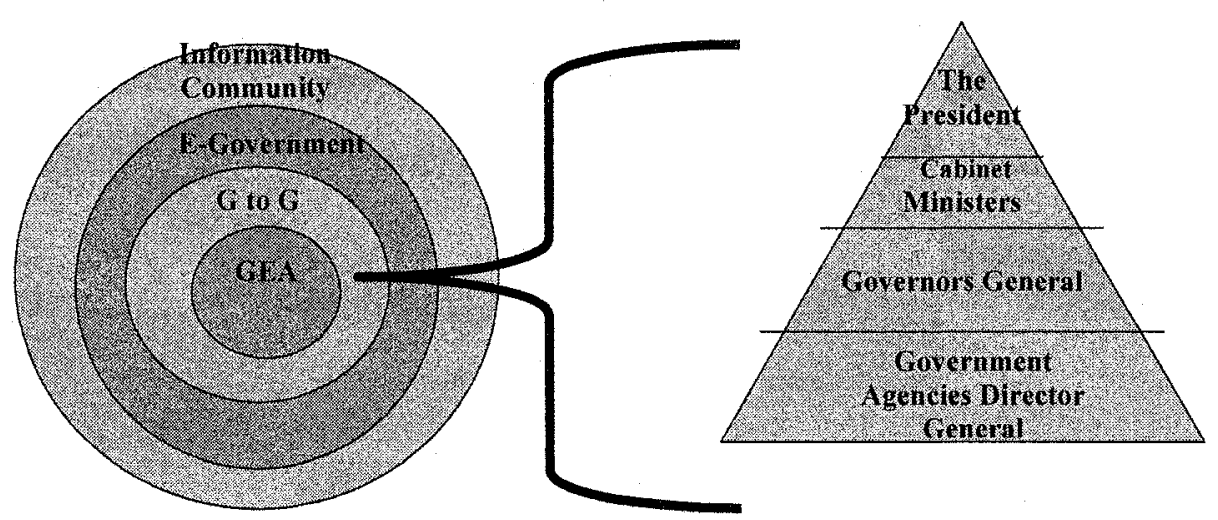

Fig. 1. Relation of G-to-G system with Information Community and e-government

It is worth explaining that GEA is the part of G-to-G which represents only the internal relationships and interactions of the government managerial body. A schematic of this concept is graphically shown in figure 2 . 


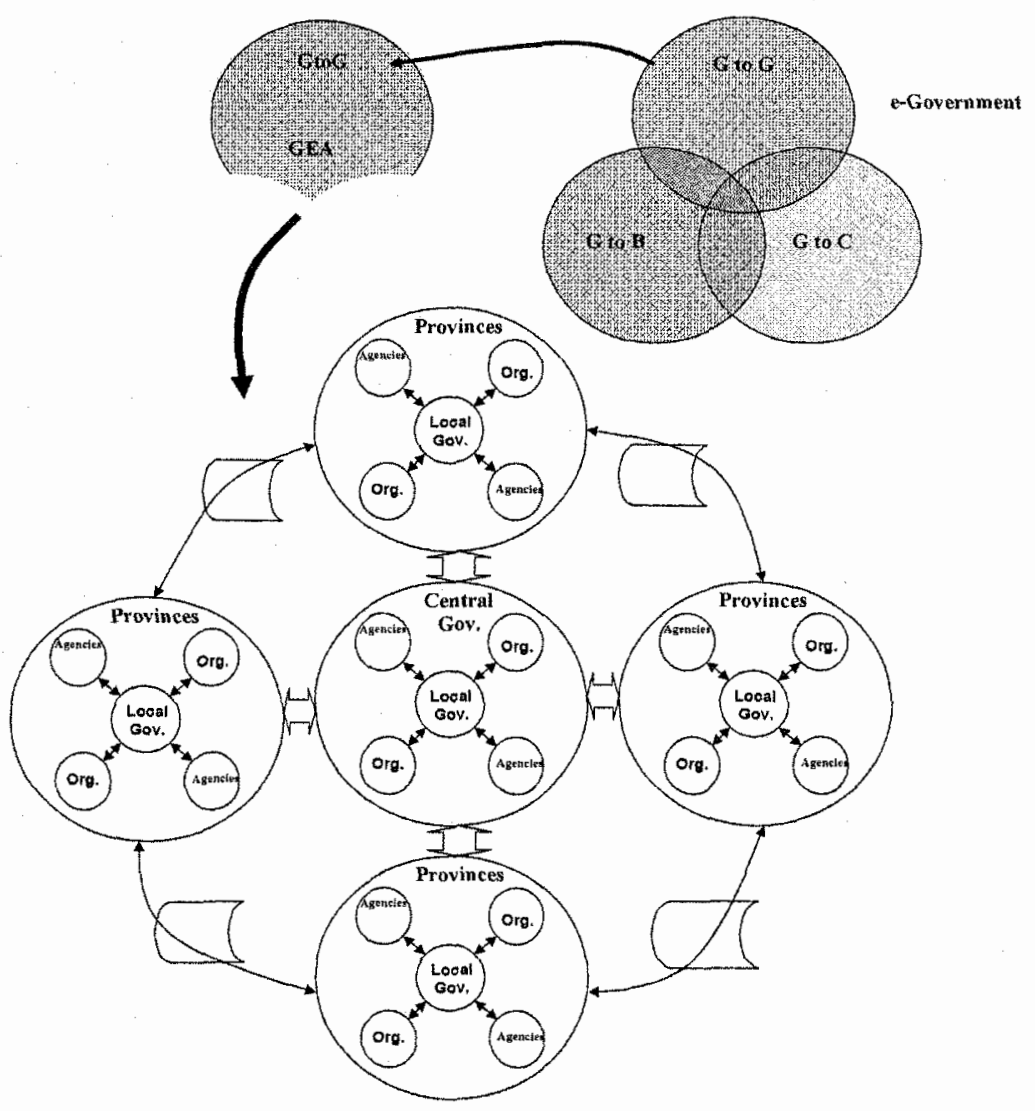

Fig. 2. E-government and its divisions including G-to-G and government Administration.

\section{GEA services and benefits}

GEA in Iran is expected to bring a unique and exceptional way for revolutionising the country's administration system. In the same time, success of this movement faces difficulties and risk. This is due to a number of reasons such as severe lack of information in general, the existing resistance against dissemination of information for security reasons, distortion of information during its flow in the administrative hierarchy, delays in access to information for decision making purposes, widespread incoherency of functions, and finally disruption of operations and tasks due to functional structure of the government. These all will lead to public dissatisfaction. 
Therefore, with respect to the presented definition and understanding of G-to-G system in Iran, and the reasons supporting its decisiveness the following services are expected from GEA.

Government integration and reduction in cycle time: The existing inefficiencies of the government agencies go much further considering the inefficient communications and interactions among agencies. The agencies are generally functioning as separate islands which not only result in weak coordination among them but also cause unbalanced growth of the government's different organisations and plans. Most of the existing dissatisfaction of the government customers is seemingly caused by inefficient government processes. For instance, a customer to receive a certain service has to move through several inter-organizational inefficient and inconvenient processes. GEA will bring about faster communications among the government agencies. This will also have considerable effects on the quality of services through creating virtual connections between agencies and improving interorganizational processes. Also since paper work forms a considerable part of the government operations, GEA can have a serious effect on this matter and remarkably improve the efficiency and effectiveness of agencies.

Government's employees involvement: The organisational structure of the government and it management has created some kind of gap between top and middle level management with skilled and expert human resources. A feeling of being treated as marginal and not considered or involved in the process of decisionmaking exists within the expert body of the government especially those working out of the capital. GEA can help remedy this feeling and establish a cooperative atmosphere through providing access channels for experts and skilled employees to the policy-makers and vice versa.

A potential for sharing experiences: Considerable expertise, talents and experience exist inside the government which are not being utilised properly. This purpose is attainable through sharing, aggregating, organising, and directing the existing potentials. This, however, would not be achieved with the very slow and imperceptible current operations of the government agencies. GEA can play an effective role in managing the existing knowledge and expertise within the country through compiling and sharing experiences. In effect it can, in long term, lead to a positive environment of competition among agencies for better utilisation of their human resources.

Preparing grounds for fundamental improvement of government in a bottom-up process: Previous experiences in improving the administrative systems of the country through top-down approaches have by no means proven successful. However, GEA implementation, which incorporates operational processes, can therefore be used as a starting point for the government processes improvement. In this approach, GEA can be used to identify redundant and ineffective processes, and to reduce the operational complexities through a bottom-up approach. Beside that, 
achieving objectives such as renovation of the government organisations' structure are expected from GEA.

Increasing access to information and transparency of operations: GEA can considerably enhance the volume and quality of accessible information for the government. Having characteristics such as speed, security, convenience, and access of management levels to information, GEA has sufficient capabilities to facilitate management by information. This can resolve many existing administrative and operational ambiguities resulting from lack of information in the executive body of the government. This service may also assist insertion of managerial controls in various levels, and also the evaluation of individuals and agencies' performance. Facilitating further control at macro level including the development and implementation of major projects and plans in various sectors would have positive effects on achieving a balanced economic growth.

Benefiting more from decision support systems: Decision support systems are playing significant and growing role in governments' operations. These systems are basically based on information from different sources including experts. GEA assists the managers in accessing the required data and also sharing the models for supporting their decisions. This issue becomes more critical in making strategic decisions.

Improving policy making capabilities: Successful implementation of the government's recent policies in reducing the government's ownership and direct control, and instead improving its abilities in making policies require government's strict steering and overseeing. Lack of these controls could result in blocking these plans and would lead to further inefficiencies in the country's administration systems. GEA can be used as a basis for data aggregation used for monitoring the operations, and conveying comments and ideas from the bottom line of the administration to the top. The result would be policies developed based on real operational data rather than outdated reports.

A foundation for e-commerce in the country: The world-wide revolutionary developments in information technology and its extensive applications in business and trade have changed the traditional concepts, learning and methods of doing business. One of the main potentials of GEA is providing grounds for electronic trade in government sectors. The efforts made in this regard so far such as development of the Electronic Trade Law are necessary but certainly not sufficient. Developing GEA is a major step in this way to provide the required foundations and preparing the government sector to join the inevitable future form of the trade system in the country. The formation of e-commerce with the support of GEA will also be effective in the growth and expansion of IT services market in the country and development of expertise in this area. 


\section{Components of GEA}

Based on the discussions made so far, seven prime constituents could be specified for GEA: Network and hardware, content, training, security, rules, procedures and regulations, and organizing and management of the network. These components are briefly described in the following:

Network and Hardware: Establishing electronic communication between government agencies requires their willingness to join the network, and feeding and updating their information onto it. Prerequisite of the agencies to trust the network is a secure, fast, attractive and economic network. In this network the government agencies are linked to communication centres through leased private lines and the centres are also linked to other centres using private networks of optical fibres to form provincial network. One of the communication centres of each provincial network will be connected to the capital (Tehran) forming the national government network. This will enable the agencies to be positioned in provincial and subsequently the national networks. These equipment should not be considered just as a physical medium, but a means for drawing attentions toward a new wave of ITbased revolutionary programs for improving efficiency, participation in decision making, bringing effects to the administration of the country at various levels of management, and shifting organizational culture to a more active and dynamic status.

$G E A$ Content and the priorities: Expectations from government physical network would not be achieved without defining and providing its content. Based on some criteria such as; operations speed, level and volume of accessible information, security of information, and distribution of information to and from top executives to other layers, the content of GEA is divided into five groups:

1. The agencies' web pages aimed at making their information available to others.

2. Basic software such as mailer aimed at enabling the agencies to start using the network and establishing communication and interaction.

3. The decision support systems aimed at processing and analyzing the generated information and providing reports to decision makers. Systems such as IT Readiness of agencies, core processes analysis, and control room system for senior level decision makers are being developed in this context.

4. Integration systems and efforts including providing systems architectural design and integration standards.

5. Managing the government information databases and banks, and transferring them to GEA.

At the best case it would be expected that the agencies develop their own contents using approved standards. However, at the current circumstances they expect to be supplied for systems and applications by some central organizations. This indeed is in contrast with the purpose of GEA. To address this problem an extensive training program is developed to enable the agencies in learning how to 
approach, start, plan, develop, and supervise their needed systems and stay aligned with the rest of the government body.

Training: Implementing GEA, as mentioned above, will be materialized through development of contents on the bed of hard communication network built for this purpose. The content in turn includes systems and software which is anticipated having major impacts on the structure and performance of the government body. Reforming or re-engineering of the government and its agencies' processes is believed to be possible only if it is accepted and originated by the management layers of the government. Overcoming the barriers in this way and moving the existing administration to this point is a grave challenge to which training is the solution. A carefully designed plan for educating government agencies management layers has been recognized as a fundamental action to prepare them for accepting changes and encouraging them to initiate change programmes. The training programme is designed in several layers and three levels, and incorporates workshops and practical work for managers to assist them approaching reengineering of their systems while getting introduced to concepts and issues. It is expected that organisational initiatives are triggered during the training courses inside the agencies for study, change and implementation of systems leading to the realisation of GEA objectives.

Security standards, codes and guidelines: The infrastructure of GEA is physically separated from the internet. However, in order to secure the applications and data, special considerations are sought. Providing the required training for employees, making the software and databases secure, and encryption activities are a few examples of such concerns. Besides moving toward open source operating systems in long term is under consideration. Also an organization for managing this issue is introduced within the agencies, and a security officer job is defined to undertake maintenance of the network's security.

Laws, rules, regulations and procedures: Development of GEA and its components must abide by general laws, rules and regulations of the country, and also comply with international laws and regulation (accepted by Iran). The complexities associated with the plan of GEA will lead to the introduction of new business methods and relationships throughout the country for which legal support should be sought. New proposals in this regard are developed and set forward for official approval.

GEA organization and management: Reaching the above mentioned goals requires insertion of extreme measures in planning, organizing and coordinating the efforts and responsible offices and organisations in the government. However, applying these measures to a system like GEA is a critical task that is caused by several issues such as:

1. Lack of consensus among officials on the definition, mission, goals and objectives of the e-government and GEA. This has resulted in the emergence of different aims and hence directions throughout the time. This problem again roots in the fact that the e-government in Iran has emerged out of three factors 
which are needs, problems, and possibilities. These issues could become highly variable and incongruent especially in Iran where the basis of decisions and policies are priorities rather than planning. At such circumstances priorities change very often which consequently affect the decisions on resource allocation,

2. Lack of sufficient scientific capabilities and expertise in managing and administrating large IT projects,

3. Inherent complexity of systems at this level and rapid changes in technology,

4. Lack of cooperation and coordination among responsible agencies.

Therefore in order to avoid formation of a new organisation, which is a part of the government strategy, a task force, in a project organization form, is formed and supported by the First Vice President to enforce the plan and make the required coordination.

\section{GEA implementation and challenges}

Moving toward development of GEA, having three main characteristics of complexity, broad scope, and diversity, encompasses various considerations each is inherently complicated on its own while having extensive interactions with others. Theses issues, to which we can refer as obstacles in the way of GEA, spread over a wide spectrum and many of them are already pointed to in previous sections of the paper. Some key issues are highlighted in the following with a short discussion on some of them:

- Vast diversity of users with different levels of capabilities and preparedness. This makes the information need analysis difficult, hinders the move to attend more serious aspects of GEA, and in practice reduces the level of systems to ordinary applications such as Mailer.

- Difficulty of reaching a unique mindset among authorities and politicians for managing and utilising GEA.

- Broad geographical and political boundary of the government, and immense size of the administrative organisation. This factor will be a major challenge for integrating the agencies and their systems.

- Incongruent and unsupportive laws and regulations pertinent to the issue of GEA and the very slow rate of change in them.

- An unfavourable mix of politics with technology. This means that the factors that influence decisions on adoption of new technology are political oriented and hence unstable in nature. Relying on such frames would be too risky.

- Short life of the managerial posts, and frequent new appointments. This matter would add insecurity and turbulence to the administrations' environment making decisions and plans very instable and unreliable. 
- Significant costs associated with the maintenance of GEA. This element will cause trouble in budgeting the government and its agencies resources, putting the health and continuity of the networks in risk.

- Existence of a traditional attitude among agencies and their managers which is the willingness to only act upon receiving orders from higher authorities and not initiate movements.

- Extensive need for educating and training managers and employees of the government. The sizeable number of agencies and hence managers, professionals and skilled employees who relate to this project from one side, and the level of IT knowledge and literacy among them from the other side necessitates a massive training programme which is very time and resource consuming and difficult to implement.

- Low level of motivation of the government managers and employees in general due to economic and organisational problems, and in particular for the change programmes towards the new frame of GEA. This matter can have fatal effects on the plans and the efforts for implementing GEA.

- Low level of available expertise in the area of ICT in the country compared to the needs, both in terms of number of professionals, consultants and ICT companies, and also in terms of level of technology and knowledge they can provide. Development of GEA in its full potential in the country requires an army of experienced experts and professionals in the field of ICT to join forces of the government agencies and transfer the resources to required systems and software.

- Difficulty in bonding the academic and research sectors of the country with the project, and utilising their abilities in developing GEA. This matter, which is a general issue for the country in most development areas, restricts the scope of potential energy available for a major movement in transforming the administration system.

Considering the mentioned restrictions and barriers associated with the development of GEA which add to its complexities, the requirements for successful accomplishment of GEA programme in Iran are identified as follows:

Content management: GEA content requires many complex and interrelated systems which needs to be coordinated by numerous organisations and individuals. This means a vast and complicated network of activities and extensive measures in planning and management. Issues such as the architecture of the content, standards, integration, setting the priorities, motivating the managers and operational groups, system analysis and development, information and application security, updating existent applications, financial resources for new applications, and assuring full utilisation of the information are among the most important challenges.

Culture and human resources: Success in utilising GEA demands close consideration of cultural and social background of the society. Understanding existing barriers as a determinant of the society readiness for accepting system 
changes is among these considerations. Furthermore, this transformation will be in severe need for educated human resources, and the current capacity of the country in this relation is insufficient and needs swift and rigorous attention and actions by authorities.

Organizational: A well-coordinated organisational model is needed for management of GEA. This model should be accredited for leading the issue in the whole country, and should aid successful completion of various projects in many different areas. This approach should also be able to remove the organizational barriers for the operational agencies given the fact that any structural changes should be approved by Management and Budgeting Office. Designing such organization is a part of GEA technology.

Technology: Choosing the right level of technology is very important. For instance, the selected software should be highly reliable and with the possibility of further development and extension.

Financial resources: Providing financial resources for timely implementation of this plan is a vital issue in the ways of its success. Obtaining resources from a bureaucratic system, cost-benefit analysis and justification, feasibility study of different projects, initiatives, negotiation for financial support, changing priorities, and lack of enough resources are some of the challenges in this group.

Time factor: GEA is being developed in response to a number of essential requirements of the country. These needs if are not fulfilled in an acceptable time period may harm the justification and validity of the project, and affect its success. Managing the project and its implementation efforts, which is not a normal practice in administrative systems, will, therefore, be a critical challenge in achieving the objectives of GEA and G-to-G

\section{Conclusions}

Moving towards e-government is a great opportunity for Iran not only to reduce its technology/digital gap with the developed world but also to improve the performance of its administration system and achieve the targeted democratic society. In order to minimise the complexities associated with the development of e-government, a particular definition is presented for e-government in Iran considering the structural and political specifications of executive systems of the country. Based on this definition, development of Government Electronic Administration (GEA) receives the top priority from among different elements of the e-government. Moving toward this objective, which entails complicated dimensions, must be planned and implemented carefully so that the country's capabilities are utilised fully, and the least possible disturbances are caused hopefully bringing about the maximum advantage for the country. Myriad Obstacles exist in the way to achieve success in this move, removing of which needs extensive and careful actions. In particular 
transforming this movement into a national culture is a crucial aspect which needs focus on cultural elevation programmes and major training programmes. Participation of universities and research centres in this national movement can significantly support and even guarantee its success.

\section{References:}

1. S.D. Hwang., Y. Choi and S.-H. Myeong, Electronic Government in South Korea, Government Information Quarterly, 16(3), 1999, pp. 277-285.

2. C.D. Slaton, T.F. Becker, Designing Electronic Government Around the World, Policy Development in the USA, Singapore, and Australia, The EDI Law Review, 7(4), 2000, pp. 122-216.

3. M.A. Wimmer, A European perspective towards one-stop government: the eGOV project, Electronic Commerce Research and Applications, 1(1), 2002, pp 92-103.

4. G. Jenkins, Observations from the trenches of Electronic Government, Ubiquity, an ACM IT magazine forume, http://www.acm,org/ubiquity/views/g_jenkins_1.html.

5. Junhura. ZHANG, A Critical Review of the Development of Chinese $e-$ Government, (Perspectives, 3(7), 2002).

6. T. L. Whitson, L. Davis, Best practices in electronic government: comprehensive electronic information dissemination for science and technology, Government Information Quarterly 18, 2001, pp.79-91.

7. D. Tapscott, Digital Economy, McGraw-Hill, (New York, 1996).

8. D. Nadler, M. Tushman, Competing By Design: The Power of Organizational Architecture, (Oxford Univ. Press, New York, 1997).

9. M.H. Sprecher, Racing to e-government: using the Internet for citizen service delivery, Government Finance Review 16 (5), 2000, pp. 21- 22.

10. J. Burn, G. Robins, Moving towards e-government: a case study of organizational change processes, Logistics Information Management, Volume 16, Number 1, 2003. pp. 25-35.

11. D. Luling, Taking it online: anyway, anyplace, anytime Tennessee anytime, The Journal of Government Financial Management, Vol. 50, Summer, 2001, pp. 42-9. 
12. G. Aicholzer, R. Schmutzer, Organizational challenges to the development of electronic government, Proceedings from $1 I^{\text {th }}$ International Workshop on Database and Expert Systems Applications, Springer, IEEE, New York, 2000, pp. 379-383.

13. NZ E-Commerce Strategy, E-commerce: building the strategy for New Zealand, 2000 , available at: www.med.govt.nz/consumer/elcom/strategy/index.html.

14. E-Government Strategy, available at: www.whitehouse.gov/omb/inforeg/ egovstrategy.pdf. 